\title{
Research on Disney's 3D Animation <Wreck-It Ralph>'s Style, Layout Pipeline, and Camera Capture System
}

\author{
Jiwon Paik ${ }^{\dagger}$, Cheeyong $\mathrm{Kim}^{++}$
}

\begin{abstract}
Disney Animation has showed not only artistic excellence but also technological innovations through a lot of animation films that they released. Especially with the unique concept that free-willed game characters dive into different worlds of games in animation film, $<$ Wreck-It Ralph $>$ received both critical and commercial acclaim for its stunning visuals and outstanding CG (computer graphics) effects. The purpose of this study is to analyze different styles of game worlds, Disney's layout pipeline, and in-house camera capture system used in <Wreck-It Ralph>. This paper analyzes that three game worlds in this film such as Fix-It Felix Jr., Sugar Rush, and Hero's Duty express different styles by using appropriate character animation and camera movements. Especially Hero's Duty game which new in-house camera capture system is extensively used maximizes unseen visuals by perfectly making realistic and believable game world. Disney's newly developed in-house camera capture system, which is used in this film for the first time, allows real camera's motion and shake and real-time camera's movement and correction within animation set. Result of this study proves that this system improves directing of feature animation and enhance efficiency of the layout department's production process. Therefore, it contributes to a great extent to development of animation films' business.
\end{abstract}

Key words: Disney, Camera Capture, Layout

\section{INTRODUCTION}

Disney Animation has marked new history by showing its artistic excellence as well as technological innovations through a lot of feature animation films that they released. Especially, $<$ Wreck-It Ralph $>$ (2012) which has unique concept that freewilled game characters go across different worlds of games inside animation film. This film was nominated for the Academy Award (2013) for 'Best Animated Feature' and won the Annie Award

※ Corresponding Author: Cheeyong Kim, Address : (614714) 176 Eomgwang-no, Jin-gu, Busan S. Korea, TEL : +82-051-890-2270, FAX : +82-51-890-2265, E-mail : kimchee@deu.ac.kr

Receipt date: Sep. 16, 2013, Revision date: Oct. 15, 2013

Approval date: Oct. 30, 2013

${ }^{+}$Dept. of Animation \& Motion Graphics, Division of Design \& Animation, Daejeon Univ.

(E-mail: jiwon.paik@gmail.com )

${ }^{++}$Dept. of Visual Information Engineering, Dong-Eui Univ.
(2013) for 'Best Animated Feature' and it received both critical and commercial acclaim for its stunning and outstanding visuals.

The purpose of this study is to analyze different styles of game worlds, Disney's layout pipeline, and in-house camera capture system used in $<$ Wreck-It Ralph $>$. This film uses camera movements and character animation appropriately to express unique styles of three different worlds; simplified style in Fix-It Felix Jr., classical Disney style in Sugar Rush, and futuristic shooting game style in Hero's Duty.

Disney Animation developed new in-house camera capture system, which is similar to live-action film's one, and made a great use of the system in first person point-of-view shooting game, Hero's Duty. This camera capture system not only makes realistic camera movements and shake possible, but also enhances efficiency of layout production process to a great extent. 
This study is not the first paper to analyze styles of animation film or camera system at Disney. Jae-Yoon Park's paper "Disney Feature-length Drawn Animation Since Using Digital Technology" [1] analyzes development of Disney's camera systems such as multi-plane camera, CAPS, and Deep Canvas. This study differs from the former study in that the former one focuses mainly on camera technology that Disney developed before year 2000 and applied to 2D animations, whereas this paper focuses on the camera system used from 3D animation <Bolt> (2008) to the latest <Wreck-It Ralph> (2012).

Jong-Hyeok Ahn's paper "Analysis of Animation <How To Train Your Dragon>[2]", also gives in-depth analysis on the contents and format-layout, camera technique-of Dreamworks' feature animation <How to Train Your Dragon>. This study differs from the former one in that, based on the researcher's work experiences at Disney Animation, this study analyzes the style of $<$ Wreck-It Ralph>, Disney's layout production pipeline, and new in-house camera capture system.

In following chapters, this paper will analyze styles of the game worlds featured in this film, character animation and camera movement used, Disney's layout production process, and newly developed camera capture system.

\section{STYLES OF GAME WORLDS IN $<$ WRECK-IT RALPH $>$}

It is very common that after feature animation film is released, game related to its content is released. However, in <Wreck-It Ralph $>$, the complex and unique game worlds exists in $3 \mathrm{D}$ animation and free-willed characters in each world dive into different worlds of games inside animation. This kind of unique attempt was never tried before.

This film features three different game worlds which are Fix-It Felix Jr., a 8-bit arcade game, Sugar Rush, a racing game, and Hero's Duty, a re- alistic shooting game. First of all, Fix-It Felix Jr. is a 8 -bit pixel game where characters can only move in either vertical or diagonal way like most of the games in 1980s. On the other hand, Sugar Rush is similar to Nintendo's Mario Kart, very popular game of the 1990s. It's like classical Disney Animation and world consists of vivid and delicious-looking desserts. In order to express cute and lovely atmosphere, the world is pastel-tone colored, bright, and rounded. Whereas two games listed above are close to cartoon styles, Hero's Duty, first person point-of-view shooting game is much closer to realism. This game has an atmosphere which is similar to Microsoft's popular game, Halo's, and has lots of exciting action scenes.

$<$ Wreck-It Ralph $>$ features more than 180 char- $^{-}$ acters and this number is three times more than the average number of typical Disney feature animation's characters[3]. Such characters and camera movements were all expressed carefully in order to show styles of different game worlds. First of all, in Fix-It Felix Jr., characters' movements is limited intentionally so that movement looks overly simplified while in classical cartoon type Sugar Rush, characters animation style is similar to traditional, classical, and lovely Disney animation style. In a shooting game, Hero's Duty, sequences seem to be filmed by a hand-held camera, with lots of dramatic and rough camera movements, to add realism to the film. The fast movements of the crowd and diverse action scenes seen in Hero's Duty makes Disney's new camera capture system more prominent.

So far, this paper analyzed three different game world styles of $<$ Wreck-It Ralph $>$. In order to fully create different characteristics and visuals of these worlds, Disney developed a couple of new technologies such as camera capture system, dRig[4] character technology, and new lighting technology. Among these, this paper will focus on the camera capture system and layout department's production pipeline related to it. 


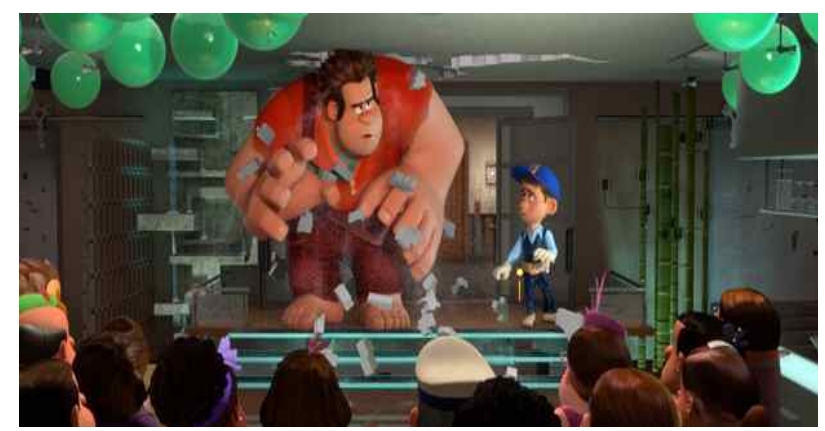

(a)

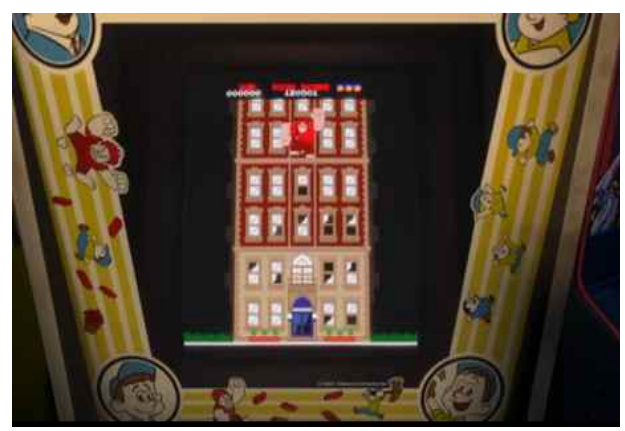

(b)

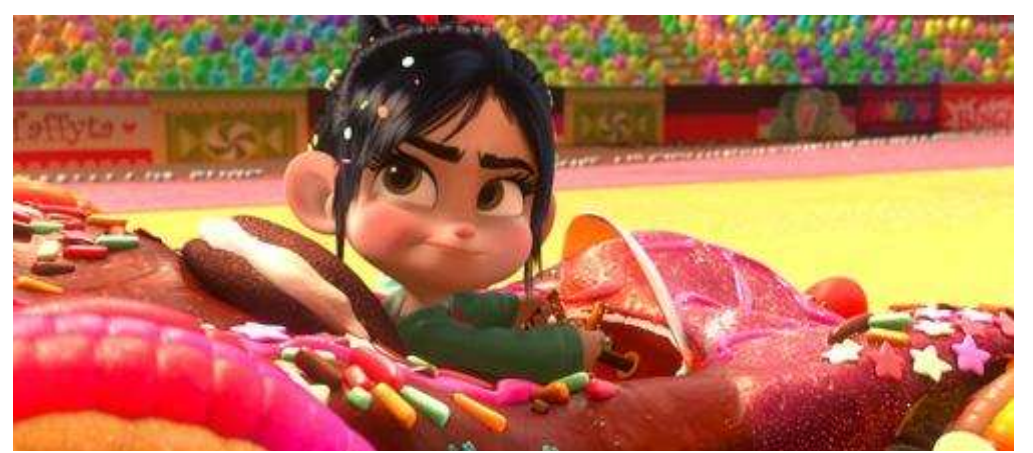

(c)

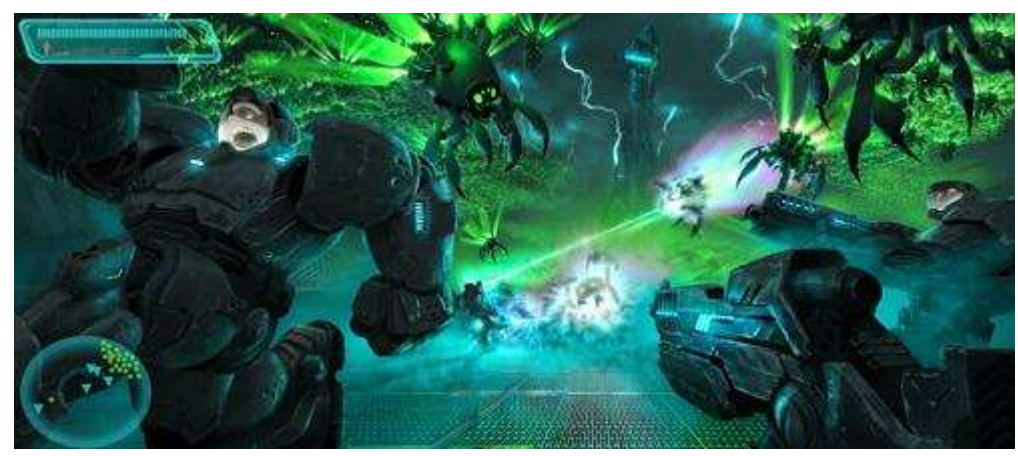

(d)

Fig. 1. (a) Ralph and Felix, the game heroes of Fix-It Felix Jr. Game[5] (b) Character and camera's movement, intentionally limited in 8 bit game world[5] (c) Lovely styles in Sugar Rush Game[6] (d) Fast and rough camera movement in chasing scene of Hero's Duty Game[7]

\section{DISNEY LAYOUT DEPARTMENT'S PIPELINE}

In 3D animation, layout department is similar to cinematography team of live-action film. It is first department in production which does pre--visualization[8] and creates 3D shots from 2D storyboard.

Disney's layout department consists of two groups: first group is 'Layout'. It arranges each element and background, locates the camera and its movement, and animate character roughly (a.k.a blocking). The second group is 'Layout Finaling'. It $\mathrm{re}^{-}$checks camera animation after animation $\mathrm{de}^{-}$ partment publishes[9] its work, checks whether all elements of a scene through a camera look fine, and exchanges layout models with actual production models to make a set.

Director has to make a plan for every single shot and give directions of his or her expectation to layout artists[10]. Since diverse experiments are required in layout stage to save downstream departments' time, layout artist sometimes show a 
Table 1. Styles of Game Worlds in <Wreck-It Ralph>

\begin{tabular}{|c|c|c|c|}
\hline Game Name & Fix-It Felix Jr. & Sugar Rush & Hero's Duty \\
\hline \hline Game Type & 8-bit Arcade Game & Cart Racing Game & $\begin{array}{c}\text { First person point-of- view } \\
\text { Shooting Game }\end{array}$ \\
\hline Style & 8-bit pixel game popular in 1980s & $\begin{array}{c}\text { popular in 90s, } \\
\text { Similar to Mario }\end{array}$ & $\begin{array}{c}\text { Realistic, a lot of actions and } \\
\text { thrills, Similar to Halo }\end{array}$ \\
\hline $\begin{array}{c}\text { Character and } \\
\text { Camera } \\
\text { Animation }\end{array}$ & $\begin{array}{c}\text { Intentionally limited character } \\
\text { movements. Simple camera } \\
\text { movements }\end{array}$ & $\begin{array}{c}\text { Old, lovely Disney } \\
\text { animation style }\end{array}$ & $\begin{array}{c}\text { Realistic character animation. } \\
\text { Dramatic, rough, and hand-held } \\
\text { camera movements }\end{array}$ \\
\hline
\end{tabular}

couple of shots using different camera animation so that director can choose the best shot among them[11]. In some cases, elements in a scene are added or removed after director reviews works from layout department which influences downstream departments' works. (Figure. 2(b))

In conclusion, layout department creates 3D animatic which not only include precise camera direction, audio, length of a shot, timing, staging[12] which has locations of character and props and scene composition, but also cover the overall control of the tone, and character's eye contacts and even moods.

As seen in production pipeline in [Figure 2](a), finished work from layout department is handed down in consideration with importance of shot's effects. If the effects leads and influences an entire shot (for example, a space shuttle in a scene catches on fire and falls), work is then handed down to the ruff effect department first. If not, work is passed to animation department. After animation department publishes its work, then it is passed to layout finaling department. Since director manages overall flow of the work by reviewing movie files from layout department first, and since he or she might try new ideas or makes modifications to the works, layout department's work efficiency and production time are pivotal in the whole process

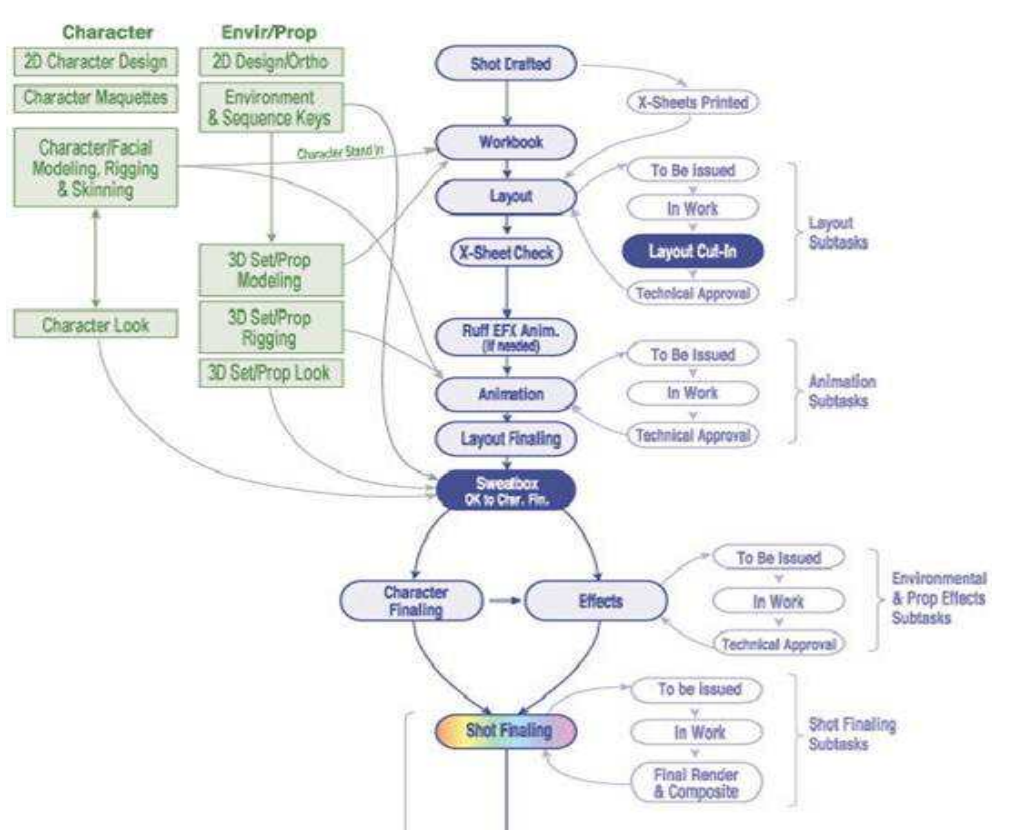

(a)

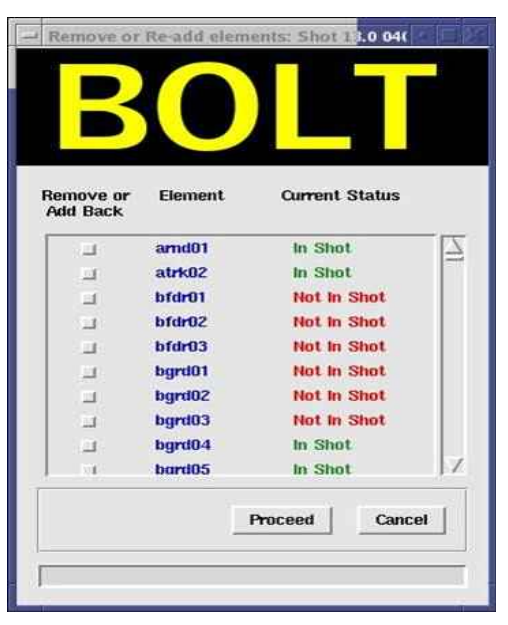

(b)

Fig. 2. (a) 'Layout' and 'Layout Finaling' dept. in Disney Animation's Production Pipeline (b) Disney Tool to add or remove characters and props. ex) <Bolt>'s 13.0/040.00 


\begin{tabular}{|c|c|c|c|c|}
\hline \multicolumn{3}{|c|}{ Frames: 66 Modlified; 2007.05:02 10:5s:40.503 } & Follows: 70.01005 .00 & Links: \\
\hline DEPT ARTIST EXT & START FINISH & ACTUAL & & NOTES \\
\hline Layout Blaisden 9149 & $04-16-07$ & $05-31-07$ & & a \\
\hline Animation & $06-01-07$ & $06-05-07$ & & - \\
\hline $\begin{array}{l}\text { Layout } \\
\text { Finaling }\end{array}$ & $06-06-07$ & $08-12.07$ & & * \\
\hline $\begin{array}{c}\text { Charader } \\
\text { Finaling }\end{array}$ & $06-14.07$ & $06-18-07$ & & e \\
\hline $\begin{array}{r}F X \\
\text { Lighting }\end{array}$ & $06-14-07$ & $06-20-07$ & & • \\
\hline$F X$ & $06-21.07$ & $06-25.07$ & & - \\
\hline $\begin{array}{r}\text { Shot } \\
\text { Finaling }\end{array}$ & $06-26-07$ & 07-03-07 & & - \\
\hline
\end{tabular}

Fig. 3. Disney's tool to show production date of each dept.'s work.

of animation pipeline.

Through in-house web-based database tool, Disney lets all the artists to see on-going and finished shots from each department's. [Figure 3] shows production records from different departments of <Meet the Robinsons $>$ (2007)'s shot 70.0/605.00. As we see production dates of each department, if works from layout and layout finaling department are delayed, then it affects shot start dates of all the downstream departments such as effects, animation, character finaling, and shot finaling.
When layout artist finishes a shot, it goes through publish process and are saved onto Disney's database, and follow-up departments use a program, as seen in [Figure 4], to download previous department's work in order to create new shot. The reason for not using existing Maya scene file from previous department, but creating new file, is to prevent passing along useless elements, garbages, possible problems in a scene which may cause Maya crashes, unusual lateness, and file corruption.

In order to enhance efficiency of layout $\mathrm{de}^{-}$ partment's pipeline and express shots more effectively, Disney developed in-house camera capture system. Next chapter analyzes camera system used prior to <Wreck-It Ralph $>$ and newly devel ${ }^{-}$ oped camera capture system.

\section{DISNEY'S CAMERA SYSTEM}

\subsection{Camera System prior to <Wreck-It Ralph>}

As Jae-Yoon Park's paper "A Study on Disney

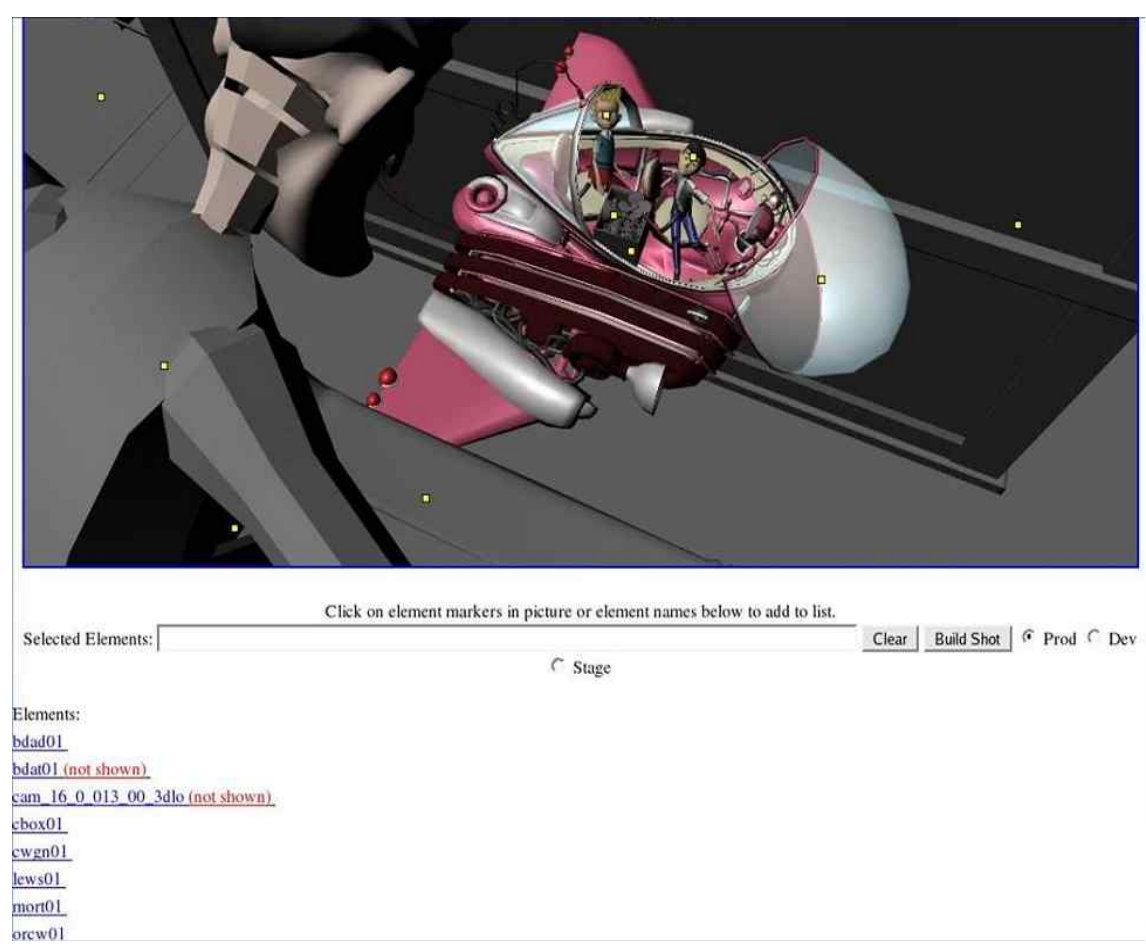

Fig. 4. Disney's program letting users select elements needed to create new Maya shots. 

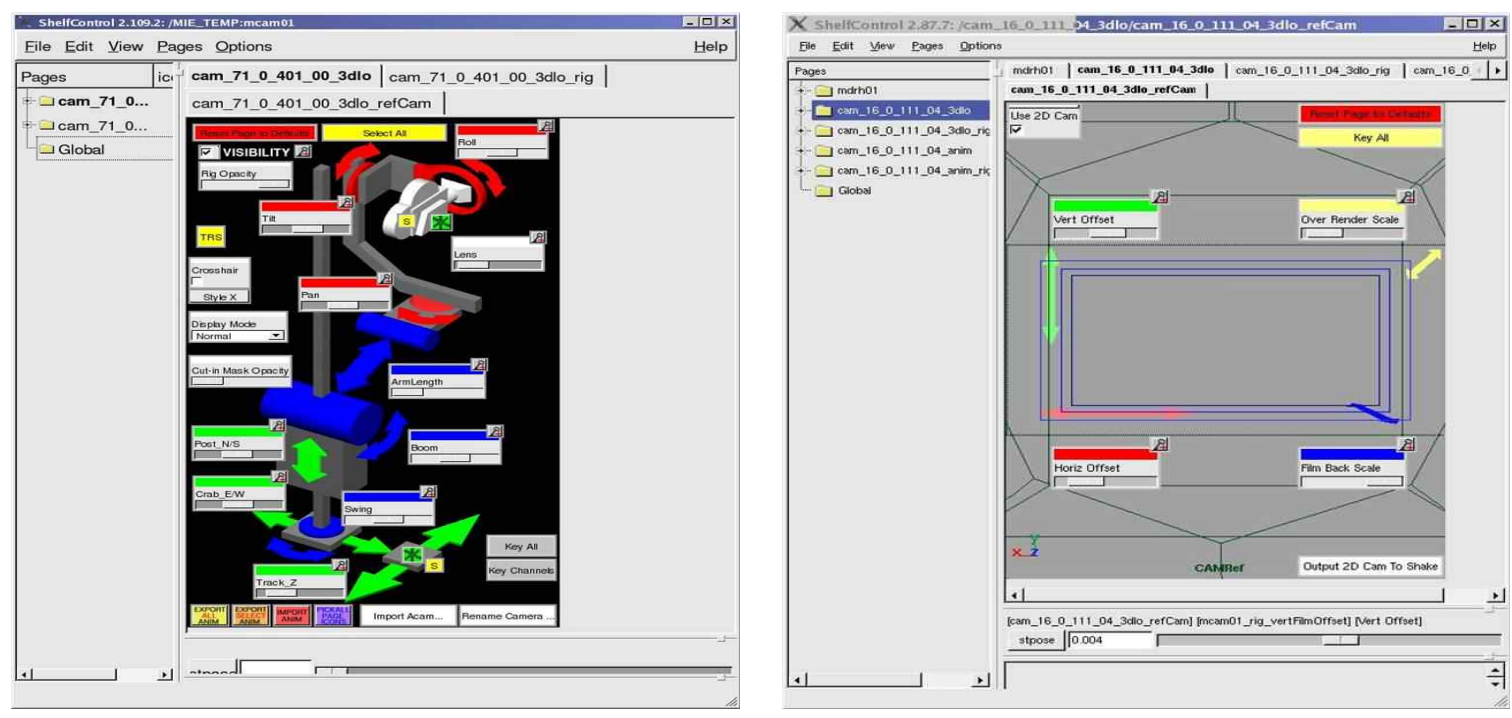

Fig. 5. Camera System developed by Disney prior to <Wreck-It Ralph>.

Feature-length Drawn Animation Since Using Digital Technology" mentions, Disney has developed multiplane camera technology which gave a visual depth in film in 1930s films such as $<$ The Snow White $>$ and $<$ Bambi $>$. In 1990s, Disney introduced CAPS (Computer Animation Production System) to digitally systemize not only camera but 2D animation, releasing The Little Mermaid (1989), Beauty and the Beast (1991), Aladdin (1992), and Lion King (1994) and then in making Tarzan (1999), Disney introduced Deep Canvas system so that cameras could hover between different layers of digital paintings[1].

In 3D animation <Bolt> (2008), Disney used a program which not only has Maya's camera's features but extra features are added as seen in [Figure 5]. This system is developed using animation toolkit called QT and program uses actual movements of camera rig such as roll, pan, tilt, boom, swing, shake, and has 2D camera features inside 3D camera. However, there is still limitation that artists can use this system only inside Maya.

\subsection{Camera Capture System in $<$ Wreck-It Ralph>}

In contrast to the previous method, in which camera movements could only be controlled within
Maya, new method used in <Wreck-It Ralph $>$ first introduces camera capture system, in which an artist can handle real camera with a marker or steer the camera on a tripod in capture studio. Since this system is linked to Disney's production process which is based on Maya, when user changes real camera, camera in Maya scene is changed instantly. Users can see result of Maya scenes from changed camera view in various screens mounted in camera capture studio's walls as seen in [Figure 6].

As in live-action filming, where director changes camera movements by changing characters, backgrounds, or props' location first, this camera capture studio allows its users to do the same, with much ease and speed. In the world of animation, it's 24 frames per second. Expressing real and detailed movements of camera requires an experienced layout artist to insert numerous keys in proper place appropriately in 3D computer program such as Maya. By using new system, however, the user can use real camera, conveying the real and vivid details of movement at ease.

Furthermore, even artists who are not familiar with Maya, such as storywriters, 2D animators, or artists from live-action film can use this system at ease and show result. Especially directors can 

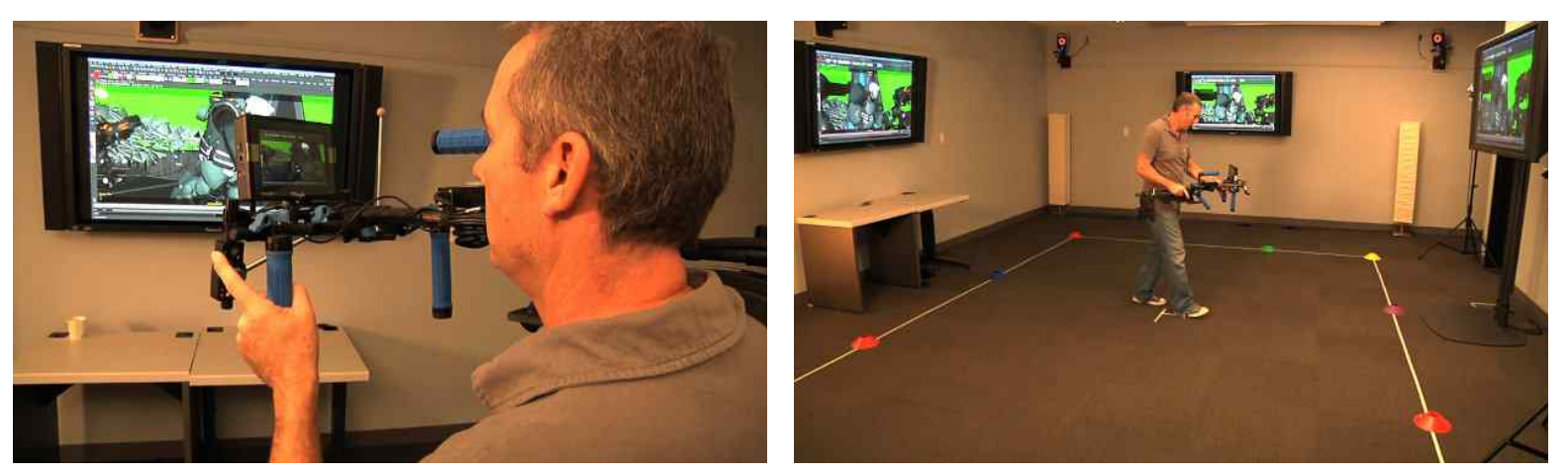

Fig. 6. A layout artist at Disney is using new Camera Capture System [13].

Table 2. Steps using Camera Capture System

\begin{tabular}{|c|l|}
\hline \multicolumn{2}{|c|}{ Steps using Camera Capture System } \\
\hline \hline 1 & Review a scene that artist worked on. Or start from process No. 2 \\
\hline 2 & $\begin{array}{l}\text { Use real camera in camera capture studio, or modify its movements. } \\
\text { Re-arrange the elements in a scene, if necessary. }\end{array}$ \\
\hline 3 & Camera animation is copied into camera in Maya. \\
\hline 4 & Review modified scene in screens in the studio. If rework is needed, go to process No. 2. \\
\hline
\end{tabular}

do numerous tests and suggest a guideline for shots. Before this system is developed, after a director give orders, he or she has to wait for weeks to actually see works done by layout department. With new system, director and layout artists can control camera movements even together and modify locations of characters, backgrounds, and props at ease, discussing modification decisions on the spot[13]. Director can show shot plans in his or her mind using real camera in camera capture studio and also suggest scene structure or composition in front. These all boil down into a significant decrease in layout department's work time and communication time between director and artists as well.

This system was adequately used in all of the game worlds, but was most prominently used in the world of Hero's Duty, the world with numerous battle action scenes and thrilling chasing scenes. Detailed movements from cameraman's waist, shoulders, or even feet movement can be conveyed to the audience. As Disney presented in FMX (Film and Media Exchange) 2012 Conference[14], Disney is willing to improve this system further to let users make use of the system with convenience, and also to be able to express even the subtle movements of simulation particles.
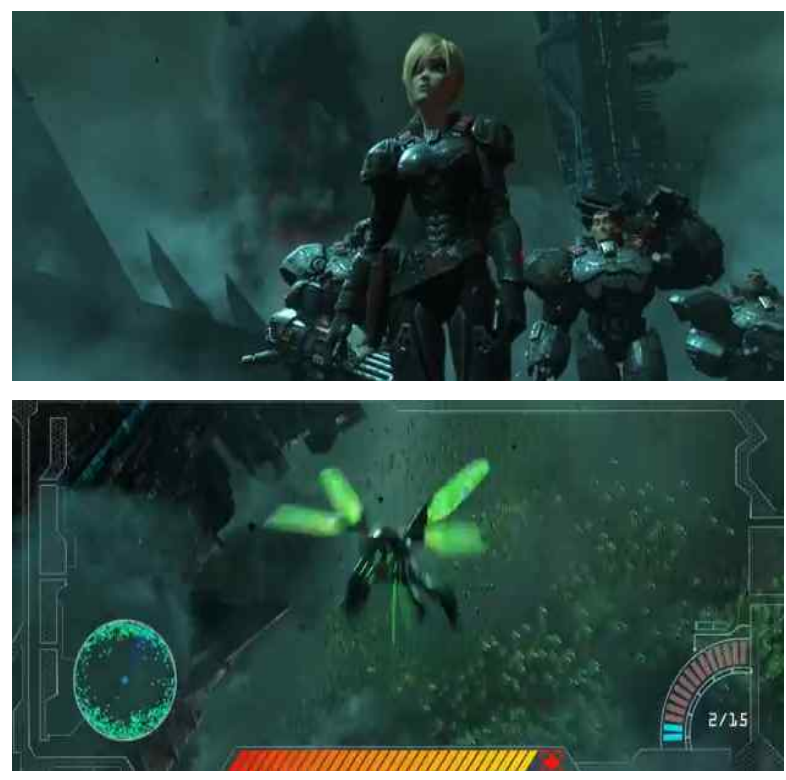

Fig. 7. Shots of game world "Hero's duty" created from camera capture system[15].

$<$ Top> Warriors' battle scene which shows radical camera movement.

$<$ Bottom> Cy-bugs' attack scene where camera movement is similar to real, handheld camera's one. 
This research proves that camera capture sys ${ }^{-}$ tem has advantages of live-action filming method and let pre-visualization possible inside camera capture studio. By letting director and artists collaborate in the studio to change camera movements and staging in a virtual set even in real time, it increases efficiency layout department's production process to a great extent. When compared to the traditional animation studio where layout departments work with cameras within a computer pro- $^{-}$ gram only such as Maya, development of new system is fundamental change and noteworthy innovation.

\section{CONCLUSION}

This study analyzes unique styles of game worlds from $<$ Wreck-It Ralph $>$, layout pipeline of Disney to fully express these worlds, and new camera capture system developed for this film. $<$ Wreck-It Ralph $>$ is the most complex 3D feature animation film ever made by Disney. It is a masterpiece that makes excellent use of character animation and camera movements to show unique characteristics of each game world. Even with more than 180 characters, there is hardly a fault in the overall structure and visuals.

John Lasseter, the CCO of Disney and Pixar Studios, says 'The art challenges the technology, and the technology inspires the art'. This film is combination of Disney's indispensable know-hows from long time experience and revolutionary innovation to create outstanding visuals. Especially Hero's duty which new in-house camera capture system is extensively used maximizes unseen vis ${ }^{-}$ uals by perfectly making the realistic and believable game worlds. This study shows that this system allows real camera's motion and shake and make real-time camera's movement and correction within animation set possible. After analysis, we can conclude that new camera capture system improves directing of feature animation and enhances efficiency of the layout department's pipeline.

Comparing to the way that how layout is done in traditional animation pipeline where artists work with cameras within a computer program only such as Maya, new system is fundamental change and innovation. Therefore, this system contributes to a great extent to animation films business as a whole.

Future research will focus on analyzing production pipeline studios in South Korea and major studios in other countries such as US and find innovative system such as camera capture system. Domestic studios need a better system and pipeline as Sung-Kyu Choi's paper "A Study on Technology Trends of CG in Visual Effects and Suggestion[16]" also points it out since domestic studios have less budget, limited number of artists, and shorter time period comparing to major studios in foreign countries. Therefore, the goal of future study is to adjust new system to domestic industry field and propose it to provide a stepping stone for the progress of the animation films' business in South Korea.

\section{REFERENCES}

[1] Jae-Yoon Park, "A Study on Disney Featurelength Drawn Animation Since Using Digital Technology," Cartoon \& Animation Studies, Vol. 26, pp. 57-78, 2012.

[2] Jong-Hyeok Ahn, "Analysis of Animation $<$ How To Train Your Dragon>", Journal of Korea Contents Association, Vol. 11, No. 12, pp. 162-170, 2011.

[3] Wreck-It Ralph Production Notes, http://www. visualhollywood.com/movies_2012/wreck-it_ ralph/notes.pdf, 2013.

[4] Greg Smith, Mark McLaughlin, Andy Lin, Even Goldberg, and Frank Hanner, "dRig: An Artist-Friendly, Object-Oriented Approach to Rig Building," Siggraph 2012, 2012.

[5] Walt Disney Animation Studio's "Wreck-It 
Ralph" Trailer Page, http://www.disneyanimation. com/projects/wreckitralph, 2012.

[ 6] Wreck-It Ralph' Inspires Technological Innovations at Walt Disney Animation Studios, https://thewaltdisneycompany.com/blog/wre ck-it-ralph-inspires-technological-innovations-walt-disney-animation-studios, 2012.

[7] Maggie Malone and Jennifer Lee, The Art of Wreck-It Ralph, Chronicle Books, San Francisco, pp. 50-51, 2012.

[8] Charles Finance and Susan Zwerman, The Visual Effects Producer, Focal Press, Boston, 2010.

[ 9] Mayur Patel, The Digital Visual Effects Studio, Clock and Flame Studios, Toronto, 2009.

[10] Catherine Winder, Producing Animation, Focal Press, Boston, 2011.

[11] Jeremy Bern, Digital Lighting and Rendering, New Riders Press, Indianapolis, 2006.

[12] Jeremy Cantor and Pepe Valencia, Inspired 3D Short Film Production, Thomson, Boston, 2005.

[13] All The World's a Virtual Stage in Disney's New Camera Capture System, AWN, http:// www.awn.com/articles/technology/all-theworld-a-virtual-stage-in-disneys-newcamera-capture--system, 2013.

[14] Evan Goldberg, "Camera Capture for "Wreckit Ralph": Introducing Capture Technology into a Feature Animation Pipeline", FMX 2012, 2012.

[15] Walt Disney Animation Studio's "Wreck-It Ralph" Trailer Page, http://www. disneyanimation.com/projects/wreckitralph, 2012.

[16] Sung-Kyu Choi, "A Study on Technology Trends of CG in Visual Effects and Suggestion", Journal of Korea Multimedia Society, Vol. 12, No. 4, pp. 591-598, 2009.

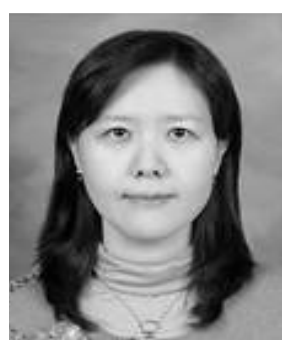

Jiwon Paik

Prof. Jiwon Paik received her bachelor's degree in Computer Science, Boston College. Then she received her master's $\mathrm{de}^{-}$ gree in Computer and Information Science, University of Pennsylvania. She worked at Walt Disney Animation Studio as a software engineer (2000 2003). She also worked at Sony Pictures Imageworks as a technical director (2003 2004) and then moved back to Walt Disney Animation Studio and worked as a technical director (2004 2007). From 2008 to Jan. 2010, She worked as a visiting professor at KAIST, Graduate School of Culture Technology. Currently she is an assistant professor at Deajeon University, Dept. of Animation \& Motion Graphics since 2010, March. Her research interest is 3D Animation, Visual Effects, and Digital Contents.

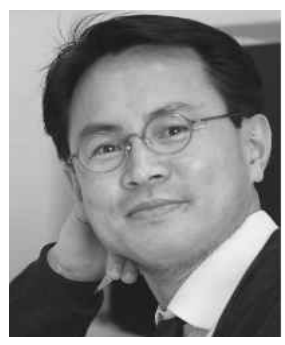

\section{Cheeyong Kim}

Associate Professor Kim Cheeyong acquired a bachelor's degree in Physics and a master's degree in Computational Physics from Inje University. He worked as a researcher at the Institute of Computer Design Education of Inje University from 1991 to 1999, full time lecturer of Department of Information and Communications at Busan Info-Tech College from 2000 to 2002, assistant professor of Department of Digital design at Dongseo University from 2003 to 2005, visiting professor at Oxford University in 2007, and visiting professor of the Digital Clothing Center at Seoul National University in 2012. Currently, he is an associate professor of Department of Visual Information Engineering at Dong-Eui University. Prof. Kim held private $\mathrm{ex}^{-}$ hibitions four times both in Seoul and China and joined over 200 international group exhibitions. With his deep interest in 3D Animation, Fractal \& Chaos Design, Computational Simulation, and 3D virtual fashion fitting, Kim passionately researches and gives presentations on the convergence of film and engineering. 